DOI: 10.30842/ielcp2306901524339

Larissa Naiditsch

(The Hebrew University of Jerusalem)

\title{
,HAUS' UND ,HOF' IN DEN DEUTSCHEN NSELMUNDARTEN DER UKRAINE IN DEN 1920er JAHREN NACH DEM ARCHIV VON VIKTOR SCHIRMUNSKI
}

\section{'House' and 'yard' in German "insular" dialects in Ukraine in 1920ies according to the archive of V. M. Žirmunsky}

The purpose of this paper is to continue studving Viktor Maximovich Žirmunsky's dialectological archive in St. Petersburg. The archive contains data about the dialects of Russian Germans who lived in the southern regions of the USSR in the 1920ies. Žirmunsky, his students, and colleagues were studying the "insular" German dialects in the Crimean peninsula, Ukraine, and Transcaucasia. They planned to publish the dialectal vocabulary of a farmer, using, among other methods, the vocabulary surveys. This work was interrupted by the tragic events of the 1930ies and 1940ies which resulted in the persecution of ethnic Germans in the USSR.

The paper examines the archive's unpublished dialectological materials, focusing on the questionnaires filled out by respondents in the 1920ies. The analysis of the lexical units 'house' and 'yard' is presented.

Keywords: German dialectology, German insular dialects in the USSR, dialectological archive of Viktor Maximovich Žirmunsky, dialectal vocabulary.

Л. Э. Найдич

(Еврейский университет в Иерусалиме)

\section{«Дом» и «двор» в немецких островных диалектах Украины в 1920-х годах по архиву Виктора Максимовича Жирмунского}

Статья продолжает изучение диалектологического архива Виктора Максимовича Жирмунского в Санкт-Петербурге, где хранятся материалы, касающиеся говоров российских немцев на юге СССР. Жирмунский и его ученики занимались в 1920-е годы изучением островных диалектов российских немцев. Они готовили материалы для диалектного словаря крестьянского хозяйства, используя, в том числе, словарные анкеты. Собранный лексический материал немецких островных диалектов, т. е. заполненные информантами в 1920-х гг. анкеты, остаются неопубликованными. В статье рассмотрена диалектная лексика на тему 'дом' и 'двор' из архива.

Ключевые слова: немецкая диалектология, немецкие островные диалекты на территории СССР, диалектологичекий архив В. М. Жирмунского, диалектная лексика. 


\section{Einleitung}

Der vorliegende Aufsatz setzt die Erschließung des dialektologischen Archivs des prominenten Philologen Viktor Schirmunski (Viktor Maximowitsch Žirmunskij) fort ${ }^{1}$. Bekanntlich wandte sich dieser Gelehrte, der sich damals schon einen Namen durch seine Studien der deutschen Literatur, der Verstheorie und der zeitgenössischen Lyrik gemacht hatte, in den 1920er Jahren der Erforschung der deutschen Sprachinseln in der UdSSR zu. In der zweiten Hälfte der 1920er Jahre unternahm er zusammen mit seinen Mitarbeitern und Schülern Studienreisen in die deutschen Kolonien auf der Krim, im Gebiet Odessa, in Transkaukasien, wie auch in dem Gebiet Leningrad, um dialektologische und Folklorematerialien zu sammeln (Zinder 1998, Svetozarova 2013).

Gleichzeitig wurden Fragebögen in die deutschen Siedlungen geschickt, die dort mit Hilfe der Dorflehrer ausgefüllt und zurückgeschickt wurden, was der indirekten Methodik in der Dialektologie entsprach. Die Fragebögen enthielten die in der deutschen Dialektologie traditionellen Wenkers Sätze. Da diese Testsätze vor allem für die Ermittlung der phonetischen Besonderheiten der Dialekte, aber auch (in geringerem Ausmaß) ihrer grammatischen Züge dienten, ergab sich die Notwendigkeit, weitere, auch lexikologisch orientierte Fragen zusammenzustellen. Schirmunski fügte dem traditionellen Fragebogen noch eine Liste der Wörter und Wendungen hinzu. Aber auch das erwies sich als ungenügend. Als Folge dieser Erkenntnisse entstand noch ein Forschungsprojekt: ein Wörterbuch der Lexik der Bauernwirtschaft wurde geplant. Dabei mussten sowohl sprachliche, als auch volkskundliche Angaben nach dem Prinzip Wörter und Sachen fixiert werden. (Žirmunskij 1932=1976: 446). Wortgeographie interessierte den Gelehrten u. a.

1 In den Jahren 2014-2016 wurde die Erforschung des Archives im Rahmen des Projekts „Germanistische Archive in St. Petersburg. Wissenschaftliche Aufarbeitung des Archivs V. M. Schirmunski (Žirmunskij) an der Akademie der Wissenschaften“" (RFH No. 13-04-00369) durchgeführt. An dieser Forschungsarbeit nahmen Natalija Swetozarowa, Larissa Pusejkina und Larissa Naiditsch teil. Vorläufige Forschungsergebnisse siehe in Svetozarova 2013, Naiditsch, Svetozarova 2015, Naiditsch 2015, 2017, 2018. В 2014-2016 гг. исследование архива проводилось в рамках проекта РГНФ («Германистические архивы в Санкт-Петербурге. Научная обработка архива В. М. Жирмунского в СПФ АРАН»), проект № 13-04-00369, 2014-2016 гг. В исследовании принимали участие Н. Д. Светозарова, Л. Н. Пузейкина и Л. Э. Найдич. Предварительные результаты исследования изложены в публикациях Svetozarova 2013; Naiditsch, Svetozarova 2015, Naiditsch 2015, 2017, 2018. 
vom Standpunkt der Sprach- und Dialektmischungen aus, die er zuerst am Material der Phonetik untersucht hatte. "Eine große Bedeutung kommt beim Erforschen dieser Vermischungsprozesse der geographischen Lexikologie zu, mithin den Fragen, die mit der territorialen Differenzierung zu tun hat" (Žirmunskij 1933-2018: 2, 184). Dabei betonte er die Rolle des ,,volkskundlichen Wortschatzes“. Somit wurde von Schirmunski noch ein Fragebogen erarbeitet und in mehrere Kolonien geschickt. Die Studienreisen und zwei Arten der Fragebögen mussten ein umfassendes Bild des Lebens von deutschen Kolonisten liefern und die Kolonien in vollem Umfang beschreiben: aus der Perspektive der Sprache, der Folklore und der Volkskunde.

Die Lexik zum Thema Haus und Hof erforschte der Gelehrte parallel zu der entsprechenden materiellen Kultur. So richtete er seine Aufmerksamkeit u. a. auf die Bauarten der Kolonistenhäuser und auf die Hofanlage. „In den deutschen Kolonien hat die Volkskunde vor allem zur Aufgabe in unterschiedlichen Gegenden, das alte kulturelle Gut festzustellen, dass die deutschen Bauern aus ihrer früheren Heimat mitgebracht haben, und die weitere Entwicklung dieser Bauernkultur auf neuem Boden, durch den Einfluss neuer geographischer, wirtschaftlicher Verhältnisse, sowie durch Kulturaustausch mit den neuen Nachbarn zu verfolgen" (Schirmunski 1925: 45). Was die direkte Forschung betrifft, so machten der Gelehrte und seine Mitarbeiter nicht nur Aufzeichnungen und Notizen, sondern auch Grammophon-Aufnahmen der Volkslieder (Bertleff, John, Svetozarova 2018); darüber hinaus fotografierten sie Menschen, Hauseinrichtungen und Gebäude. Die meisten dieser Fotos blieben bei der Familie des Gelehrten; sie wurden erst 2016 publiziert (Žirmunskaja, Starec, Naiditsch, Svetozarova 2016).

Diese Forschungsarbeit wurde Anfang der 1930er Jahre wegen des Terrors gegen die Nationalminoritäten, unter ihnen auch gegen die Sowjetdeutschen, eingestellt. Schirmunskis Mitarbeiter mussten Repressalien erleiden. Die deutschen Siedlungen im europäischen Teil der UdSSR wurden in den 1940er Jahren komplett ausgetilgt.

Die dialektologischen Materialien, u. a. die Fragebögen für das geplante Wörterbuch der Bauernwirtschaft, liegen heute in der Sankt-Petersburger Filiale des Archivs der Akademie der Wissenschaften Russlands (Fonds 1001) und werden allmählich bearbeitet. Im Weiteren sei ein Teil der ausgefüllten Fragebögen betrachtet, und zwar derjenige, wo es sich um die Lexik zum Thema Haus und Hof handelt. Sie stammen aus den Gebieten Cherson, 
Odessa und aus den anderen deutschen Gebieten im Süden der Sowjetunion.

Die hier betrachtete Lexik gehört zum rheinfränkischen (pfälzisch-hessischen) Wortschatz. Eine nähere Analyse jeder heute schon verschollenen Ma der südlichen Kolonien ist zurzeit kaum möglich. Schirmunski stellte fest, dass die meisten Inseldialekte in der Ukraine Mischmundarten waren. Manchmal handelte es sich um fränkisch-schwäbische Dialekte, bei deren Mischung schwäbische Besonderheiten (diejenigen, die der Gelehrte Primärmerkmale genannt hat) untergegangen sind (Žirmunskij 1929 = 1976: 495-497).

\section{Haus}

\subsection{Fußboden und Zimmerdecke}

Die Bezeichnungen von Fußboden und von Zimmerdecke sind in den lexikalischen Fragebögen von Schirmunski unter 30 und 31. angebracht. Vgl. folgende Belege:

Tabelle 1. Bezeichnungen für Fußboden und Zimmerdecke in einzelnen Kolonien

\begin{tabular}{|l|l|l|l|}
\hline Standardsprache & 30 a Lehmboden & 30 b Bretterboden & 31 Zimmerdecke \\
\hline $\begin{array}{l}\text { Alexandr2, vgl. } \\
\text { auch N-Grüntal, } \\
\text { Olgino, Marxfeld, } \\
\text { Feodorowka }\end{array}$ & Erdbode & Dielbode & Bode \\
\hline Sophiental & Laimbode & Bretterbode & Spaicher, Behne \\
\hline Petropawlowka & Laimbode & Diele & Potolok, Bode \\
\hline Klein Orlow & Druckboda & Brettaboda & Owaboda \\
\hline Marjanowka & Bode & Bode & Deck \\
\hline Nov. Berezniki & Lehmbode & Fußboden & Boden, Behne \\
\hline $\begin{array}{l}\text { Neu-Dmitriewka, } \\
\text { vgl. auch } \\
\text { Klosterdorf }\end{array}$ & Erdboden & Brettboden & Boden \\
\hline Schlangendorf & Erdbode & Bretterbode & Bode \\
\hline Neu-Klostertal & Diele & Diele & Boden \\
\hline Miliardowka & Lamaboda & Dielaboda & Eschtring \\
\hline Straßburg & Boden & Dielebode & Neschtreng \\
\hline Kandel & Laimbode & Bretterbode & Eschtrich \\
\hline
\end{tabular}


Somit ist Boden als ein selbständiges Wort oder als Wortteil in den betrachteten Fragebögen am häufigsten zu treffen. Es fällt auf, dass Boden in der Bedeutung ,Zimmerdecke' gebraucht wird, was den Angaben der Dialektwörterbücher, wie auch der Etymologie des Wortes nicht entspricht. Das Wort ist seit dem 9. Jahrhundert als bodem, später boden bekannt, und zwar in der Bedeutung ,Erdoberfläche, Grund“ (Etym. 154). Auch in den modernen Maa entspricht die Bedeutung dieses Wortes der ursprünglichen und nicht derjenigen in den obigen Maa. So steht im PfWb 1. 1067 (vgl. 830): Boden „Erdboden. Fläche, auf der man steht, geht; übertr. ,sichere Grundlage", in Gebäuden ,Fußboden“". Unter 3 b ist dort die Bedeutung ,Dachboden" angegeben, die seit dem 16. Jahrhundert im Deutschen bekannt ist. Sie müsste die Quelle der Umdeutung des Wortes gewesen sein. Vgl. auch NRhW und auch Post 2000: 46, wo Bodem, Boden in pfälzischen Maa als 'ErdFußboden, unterer Teil' erscheint.

Eschtrich (Estrich) bedeutet im Deutschen nach Duden ,fugenloser Fußboden, Unterboden aus einer erhärteten Masse (insbesondere Zement) ${ }^{6}$, im Schweizerdeutschen ,Dachboden, Dachraum', in manchen Maa ,Fußboden', aber auch ,Zimmerdecke" PfWb 2: 1083, RhWb 2: 203, Post 2000: 57. Ein seltenes Beispiel ist das Wort Neschtreng mit n-Prothese aus dem unbestimmten Artikel.

Gelegentlich kommen in den Fragebögen Behn (Bühne) und Speicher als ,Zimmerdecke' vor.

Der Schluss liegt nahe, dass die Benennung der Zimmerdecke in den betrachteten Maa aus derjenigen des Dachbodens entstanden ist. Boden > Dachboden > Zimmerdecke. Dachboden war ein Vorratsraum, der sich unter dem Dach befand, was sowohl für Deutschland (PfWb 1, 1068, 6: 242), als auch für die Kolonien in der Ukraine typisch war. Schirmunski schrieb über die Häuser in den Kolonien: «unter dem Dache befindet sich der Speicher (in der Ukraine auch Bühne oder Owwenuf genannt» (Schirmunski 1930: 75). Der lexikalische Unterschied zwischen 'Decke' und 'Boden' wird oft wenn nicht durch völlig unterschiedliche Lexeme, so durch Zusammensetzungen wiedergegeben (siehe oben Tabelle 1).

\section{2. Eingang, Korridor}

Das Kolonistenhaus in der Südukraine beschrieb Schirmunski folgenderweise: «Das Haus ist dreiteilig, in der Mitte die Küche mit einer Vorstube (in der Ukraine Vorderstub, Vorderkich, Hausern oder auch Hausder genannt)» (Schirmunski 1930: 75). Zwei Fragen im Fragebogen betreffen den Vorraum des Hauses: 33: „Der mittlere 
Raum des Hauses vor der Küche“ und 34 „Der gedeckte Vorbau vor der Tür". Als Übersetzung des Ersteren finden sich in den Fragebögen die Belege: Hausern, Haustear, Haustier, das Voraus, die Vorraus, Herausgang, Hausgang, Mittelstub, Kichle, Kichln, Kariderle, Kirlander, Kolida. Der häufigste ist dabei Hausern oder das davon stammende Hauster. Das altdeutsche Wort Ern, der zweite Teil des Kompositums Haus-ern, entspricht dem mhd. er(e)n ,Fußboden, Tenne'. Nach RhWb 2, 168-169 war Ern "der grosse, mit Platten belegte Hausflur in altfränkischen Bauernhäusern, der zugleich Küche war“, nach PfWb 3, 717-718 ist „Haus-ern m., f.: Hausgang, Hausflur (im Erdgeschoß) des Bauernhauses“2. „Das Wort war schon um 1930 mancherorts nur noch den Alten geläufig $<\ldots>$ In der Form Hausdeer(e) liegt Anlehnung an Haus-deer ,Haustür" vor" (ebenda). Vgl. auch Südhess. 3: 182; RhWB 3: 360. Somit besteht kein Zweifel, dass die Kolonisten dieses Wort aus ihrem Ursprungsort in Deutschland gebracht hatten, wie auch die Art des Hausbaus, die teilweise der deutschen entsprach. Die drei letzteren angeführten Belege Kariderle, Kirlander, Kolida sind die der deutschen Sprache angepassten Entlehnungen aus dem Russischen oder aus dem Ukrainischen. Die Abwechslung $r / l$ kommt oft auch in der russischen Umgangssprache als „Fehler“" vor (/kal'idor/).

Der gedeckte Vorbau vor der Tür ist eine Besonderheit des russischen Dorfhauses. Schirmunski stellte fest: „Die Teile des Hauses, die auf die russische Art gebaut wurden, wurden durch Lehnwörter benannt. So hieß das Vorhaus in vielen russlanddeutschen Maa Kriletz (aus russ. крыльцьo-kryl'co)“" (Schirmunski 1928, 117) ${ }^{3}$. Jedoch finden wir in den Fragebögen meistens deutsche Entsprechungen, die auf russischem Boden entstanden sind: Vorlaube, Vorhaus, Vorheisl, seltener Treppendecke, Überhang, Schirm, Korridor. Ein seltenes Beispiel der Entlehnung dieses Wortes aus dem Russischen ist der Griltz (Sorotschino, Gebiet Korosten).

${ }^{2}$ Vgl. die Beschreibung des Kolonistenhauses in den Erinnerungen des Pfarrers David Weigum aus der Kolonie Ludwigstal auf der Krim (1876 1952): Mein Eltern- und Geburtshaus war das kleinste von allen Wirtshäusern (Bauernhäusern) im Dorf. <...>. Bei einem plötzlichen Gewitter drang das Wasser in die Hausehrn (den Hausflur). <...>.Die Küche mit dem Backsteinherd war durch eine dünne Wand von der Hausehrn abgetrennt. Von dort aus wurden die Stubenöfen geheizt" (Weigum s.a.: 28-29).

${ }^{3}$ Vgl. Bachmann 2015: 39, wo Hermann Bachmann, ein Mitarbeiter von Schirmunski und Teilnehmer einiger seiner Studienreisen, Vorhäuschen als einen Teil des Bauernhauses erwähnte. 


\section{3. Möbel}

Von Schirmunskis Interesse für die innere Ausstattung des Hauses als eines wichtigen Elementes der materiellen Kultur zeugen einige Fotos (Žirmunskaja, Starec, Naiditsch, Svetozarova 2016: 50, 53, 61, 67, 76). Besonders interessant und schön sind Fotos der bemalten Schränke (ebenda: 67, 76) Das Wort Schrank in den Fragebögen wurde meistens als Schank übersetzt. Das entspricht dem Pfälzischen und den anderen rheinfränkischen Maa (PfWb 5: 870, RhWb 7: 912) und wurde auch im Gebiet PetersburgLeningrad belegt (Najdič 1997: 90). Das Wort Schank war bereits im Mhd bekannt und wird als Nebenform vom Schrank betrachtet (Etym. 2004: 1240).

\section{Hof}

Dreschen war ein wichtiger Bestandteil der landwirtschaftlichen Arbeit. Hermann Bachmann, ein Mitarbeiter von Schirmunski, erzählte in seiner Reisebeschreibung, wie Schirmunskis Forschungsgruppe in die Kolonie Katharinenthal zur Zeit des Dreschens gekommen war, und beschrieb diesen Prozess ${ }^{4}$ (Bachmann 1929: 44-45, Svetozarova 2015: 61-62).

„Dreschplatz auf dem Hofe“ (Nummer 34) wurde in den Fragebögen übersetzt als: Dreschplatz, Tenn, Denn, Dreschdin, Treschtin, Dreschdenn, Dreschdiele, Trempelflur. Hier finden wir phonetische oder eher Schreibvarianten des Wortes $(d-t)$. Die Form Dreschdin entstand infolge der Reduktion des zweiten schwach betonten Wortteils. Tenne ist, fest gestampfter Lehmboden in der Scheune, er diente zum Dreschen und wurde auch Dreschtenne genannt' (PfWb 2: 216, RhWb 8: 1135, Grimm 2: 1408). Die meisten Lexeme im Fragebogen entsprechen der deutschen Regionallexik. Trempelflur kommt viel seltener als andere Belege vor; der erste Teil des Wortes stammt von dem Verb trämpeln ,stampfen'.

Der Schuppen, ,Gebäude im Hof zum Abstellen von Wagen und landwirtschaftlichen Geräten', wurde als Schuppe(n), Schopf, Schopp, Schoppel, Sirej, Saraj übersetzt. Zwei letztere stammen aus dem Russischen oder aus dem Ukrainischen.

Dass Schirmunski das Wort „Kinderschaukel“ in seinen Fragebogen aufgenommen hat, ist wohl dadurch zu erklären, dass die Benennung dieses Gegenstandes für bestimmte Gegenden in Deutschland typisch ist. Zum anderen aber waren Kinderschaukeln in Bauernhöfen oft zu sehen. Schirmunski schrieb über die Lexik der

${ }^{4}$ Vgl. auch Weigum 69-73. 
von ihm erforschten Maa: „Eine Schaukel heißt gewöhnlich gauntsch (gangsch u. ä.), dagegen behält das für die russischen Dörfer charakteristische hängende Schaukelbrett seinen russischen Namen katschell" (Schirmunski 1930: 59). Das von Schirmunski erwähnte Wort Gauntsch war nach dem vorhandenen Material in den betrachteten Maa wirklich sehr verbreitet. Auch in den Maa bei St. Petersburg - Leningrad (Kolpino, Neu-Saratowka) wurde es belegt (Najdič, 1997: 141). Wie schon erwähnt wurde, ist das Wort Kinderschaukel dialektgeographisch bezeichnend. Gauntsche, Gautsche ist für die Gegend im Süd-Osten der Pfalz typisch (PfWb: 75, 76, Karte 78). Seltener sind in den Fragebögen die folgenden Formen anzutreffen: Schockel, Schukshei, Huschel und die Lehnwörter Katschele, Karusell und Lulke. Zwei erstere gehen auf den Wortstamm schuk / schock zurück: schucken, werfen, (zum Auffangen, mit leichtem Schwung) zuwerfen', (PfWb 5: 1468); vgl. auch schocken (PfWb 5: 1399) mit derselben Bedeutung. Das Wort lulke stammt vom Ukrainischen oder vom Russischen люлька (l'ul'ka) und bedeutet dort 'Wiege'.

Tabelle 2. Beispiele der Lexik zum Thema 'Haus' und „Hof' aus den Fragebögen im Archiv Schirmunski

\begin{tabular}{|l|l|l|l|}
\hline Kolonie & $\begin{array}{l}\text { Chutor } \\
\text { Rosowka } \\
\text { Rayon } \\
\text { Cherson }\end{array}$ & $\begin{array}{l}\text { Weinau, Kreis } \\
\text { Melitopol, } \\
\text { Rayon } \\
\text { Molotschna, } \\
\text { Poststation } \\
\text { Prischib }\end{array}$ & $\begin{array}{l}\text { Neu- } \\
\text { Dmitriewka, } \\
\text { Artjemow Kreis, } \\
\text { Konstantiniwsk. } \\
\text { Rayon }\end{array}$ \\
\hline $\begin{array}{l}\text { Name des } \\
\text { Lehrers }\end{array}$ & $\begin{array}{l}\text { Joseph } \\
\text { Zaengler }\end{array}$ & $\begin{array}{l}\text { Jordan } \\
\text { Christian Fr. }\end{array}$ & Isaak Wall \\
\hline $\begin{array}{l}\text { 30 Der Fußboden } \\
\text { des Zimmers } \\
\text { пол) }\end{array}$ & Der Fußbote & Dea Fußbode & ----- \\
\hline $\begin{array}{l}\text { a) Lehmboden } \\
\text { b) Zimmerboden }\end{array}$ & $\begin{array}{l}\text { Erdboten } \\
\text { Diele }\end{array}$ & $\begin{array}{l}\text { Lehmbode } \\
\text { Brettebode }\end{array}$ & $\begin{array}{l}\text { Erdboden } \\
\text { Brettboden }\end{array}$ \\
\hline $\begin{array}{l}\text { 31 Die } \\
\text { Zimmerdecke } \\
\text { (потолок) }\end{array}$ & Bodendecke & $\begin{array}{l}\text { Dea Bode } \\
\text { (Hausbode) }\end{array}$ & Boden \\
\hline
\end{tabular}

\footnotetext{
${ }^{5}$ Weinau (Arbuzovka), heute Čapaevka, Zaporožskaja oblast', lutherisches Dort, gegründet in 1804 ( Diesendorf 2006).

${ }^{6}$ Nowo-Dmitrievka. Heute Nowodmitrovka, war Doneckaja oblast', war lutherisches Dort gegründet 1909. (Ebenda).
} 


\begin{tabular}{|l|l|l|l|}
\hline $\begin{array}{l}33 \text { Der mittlere } \\
\text { Raum des } \\
\text { Hauses vor der } \\
\text { Küche }\end{array}$ & Das Koridor & Hausern & Hausgang \\
\hline $\begin{array}{l}\text { 34 Der verdeckte } \\
\text { Vorbau vor der } \\
\text { Tür (крыльцо) }\end{array}$ & $\begin{array}{l}\text { Dreppendecke } \\
\text { Tre }\end{array}$ & Vorhaus & Vorhaus \\
\hline $\begin{array}{l}\text { Dreschplatz auf } \\
\text { dem Hofe (ток) }\end{array}$ & Dreschtenne & Die Tenn & Dreschdehn \\
\hline $\begin{array}{l}\text { 36 Der Schuppen } \\
\text { (capaй) }\end{array}$ & $\begin{array}{l}\text { Schupfen, } \\
\text { auch Schoppe }\end{array}$ & Dea Schoppe & Schuppe \\
\hline $\begin{array}{l}\text { 37 Der } \\
\text { Weingarten }\end{array}$ & Weingarten & Dea Weigaete & Weingarten \\
\hline $\begin{array}{l}\text { 38 der Schrank } \\
\text { Schank und } \\
\text { Schrank }\end{array}$ & Dea Schank & Schrank \\
\hline $\begin{array}{l}\text { 39 Die hängende } \\
\text { Kinderschaukel } \\
\text { im Garten }\end{array}$ & $\begin{array}{l}\text { Schaukel und } \\
\text { Gaunsche }\end{array}$ & Die Gaunsch & Gangsch \\
\hline
\end{tabular}

\section{Schlusswort}

Die Erschließung der im Archiv Schirmunski aufbewahrten Fragebögen zum Wortschatz der Bauernwirtschaft in den ehemaligen deutschen Kolonien der Ukraine und der Krim ist eine wichtige Aufgabe der Germanistik. Der Wortschatz zum Thema 'Haus' und ,Hof' spiegelt nicht nur sprachliche, sondern auch ethnographische Charakteristika der Russlanddeutschen der südlichen Kolonien. Die Analyse des betrachteten Wortschatzes zeigt, dass er im Großen und Ganzen den rhein-fränkischen Mundarten (pfälzischen und hessischen) entspricht; somit brachten die Mundartensprecher diese Lexik aus ihrer deutschen Heimat mit. In diesem Artikel wurde aber demonstriert, dass die alte Lexik neue Bedeutungen entwickeln kann; s. den Abschnitt über die Wörter ,Zimmerdecke' und ,Fußboden', deren Bedeutungsverschiebung nach den vorhandenen Dialektwörterbüchern keine Entsprechungen in der deutschen Metropole hat. Die Lehnwörter aus dem Russischen und dem Ukrainischen zeugen von den Kontakten mit den Nachbarvölkern. In manchen Fällen handelt es sich dabei um die Benennung der Gegenstände, die für die Kolonisten neu waren, z. B. Kryletz (solche Beispiele sind in unserem Material eher selten). Manchmal wurden alte Wörter und Wortstämme an neue 
Gegenstände und Bedingungen angepasst: Treppendecke, Vorhaus als russ. крыльцо. Dabei bediente sich die Sprache (Mundart) der deutschen Wortbildungsmittel: Präfixe, Wortkomposition.

Dank der Vollendung der Arbeit an großen Dialektwörterbüchern und der Digitalisierung und Vernetzung ihrer Ergebnisse steht uns heute eine breite Palette an Möglichkeiten zur Verfügung, um den Ursprung und die geographische Verbreitung des Wortschatzes verfolgen zu können. Die angeführten Materialien können auch zur Dialektgeographie und zur Erforschung der Sprachkontakte beitragen. Das angeführte lexikalische Material spiegelt die Lebensweise und die materielle Kultur der Russlanddeutschen in den Vorkriegsjahren wider. Schirmunski selbst betrachtete es als eine wichtige Quelle sowohl für die Sprachwissenschaft, als auch für die Volkskunde.

\section{Wörterbücher und Lexika}

Diesendorf 2006: - Diesendorf V. Die Deutschen Rußlands. Siedlungen und Siedlungsgebiete. Lexikon. ERD. Moskau 2006 http://wolgadeutsche.net/diesendorf/Ortslexikon.pdf

Etym. - Etymologisches Wörterbuch des Deutschen. Hrsg. von Wolfgang Pfeifer. Deutscher Taschenbuch Verlag. München. 7. Auflage 2004.

Grimm - Das deutsche Wörterbuch von Jakob und Wilhelm Grimm: online Wörterbuch http://dwb.uni-trier.de/de/ Letzter Zugriff 9. 04. 2020

PfWb: Pfälzisches Wörterbuch, Begründet von Ernst Christmann. Bearb. von Julius Krämer, Rudolf Post. Wiesbaden/Stuttgart, Bd. I-VI, 1965-1997. Heute auch im Internet: http://woerterbuchnetz.de/cgibin/WBNetz/wbgui_py?sigle $=$ PfWB\&mode $=$ Vernetzung\&lemid $=P$ H02132\#XPH02132 (9. 4. 2020)

Post 2000 - Post Rudolf. Kleines Pfälzisches Wörterbuch. Verlag K. F. Geißler. 2000

RhWb. - Rheinisches Wörterbuch. Josef Müller / Heinrich Dittmaier / Karl Meisen / Matthias Zender. Publikationszeitraum: 1928-1971 http://woerterbuchnetz.de/cgi-bin/WBNetz/wbgui_py?sigle=RhWB (9.4.2020)

\section{Literatur}

Bachmann 1929: Bachmann H., Durch die deutschen Kolonien des Beresaner Gebiets, Charkow, Zentralverlag.

Bertleff, John, Svetozarova 2018: Ingrid Bertleff / Eckhard John / Natalia Svetozarova, Russlanddeutsche Lieder. Geschichte - Sammlung Lebenswelten. Band 1: Liedgeschichten und Editionen. Band 2: Analysen und Quellen. Mit Beiträgen von Konstantin Azadovski und Dietmar Neutatz sowie einem Repertorium zur Sammlung Viktor Žirmunskij (Deutsches Volksliedarchiv Leningrad). Institut für Russische Literatur (Puškinskij Dom) der Russischen Akademie der Wissenschaften St. Petersburg). Albert-Ludwigs-Universität Freiburg. Essen: Verlag Klartext. (Veröffentlichungen zur Kultur und Geschichte im östlichen Europa. Band 52-1, 52-2). 
Naiditsch 2008: Naiditsch Larissa. Viktor Maksimovič Schirmunski Germanist und Mundartforscher. In: Peter Ernst (Hrsg.). Bausteine zur Wissenschaftsgeschichte von Dialektologie / Germanistischer Sprachwissenschaft im 19. und 20. Jahrhundert. Beiträge zum 2. Kongress der Internationalen Gesellschaft für Dialektologie des Deutschen, Wien, 20.-23. September 2006. Praesens Verlag, Wien, 55-74.

Naiditsch 2015: Naiditsch Larissa. Das Mennonitenplatt (Plautdietsch) nach den Fragebögen aus dem Archiv Schirmunski. Teil I. Probleme des Konsonantismus. Zeitschrift für Dialektologie und Linguistik. 2015. Bd. LXXXII, H. 3, 331-349.

Naiditsch 2017: Naiditsch Larissa. Zur Lexik im Mennonitenplatt nach dem Archiv von V. M. Schirmunski. Indo-European Linguistics and Classical Philologv. 21. Индоевропейское языкознание и классическая филология 21, 1012-1036.

Naiditsch 2018: Naiditsch Larissa. Zum Wortschatz in den deutschen Inselmundarten der Ukraine in den 1920er Jahren. Nach dem Archiv von Viktor Schirmunski. Indo-European Linguistics and Classical Philology. Индоевропейское языкознание и классическая филология 22, 919-944.

Najdič 1997: Najdič Larissa, Deutsche Bauern bei St. Petersburg Leningrad. Dialekte - Brauchtum - Folklore. Stuttgart: Franz Steiner Verlag, 1997 (ZDL Beihefte 94).

Naiditsch, Svetozarova 2015: Naiditsch L., Svetozarova N. Dialektologische Projekte von V. M. Schirmunski und die Wortkartei von L. R. Sinder. Indo-European Linguistics and Classical Philology. Индоевропейское языкознание и классическая филология. 19.

Schirmunski 1925: Schirmunski Viktor. Studien zur Volkskunde der deutschen Kolonien. Zur neuen Schule, 14-15. S. 45-57.

Schirmunski 1928: Schirmunski, Viktor. Die deutschen Kolonien in der Ukraine. Moskau : Zentral-Völkerverlag der Sowjet-Union, 1928.

Schirmunski 1930: Schirmunski, Viktor. Volkskundliche Forschungen in den deutschen Siedlungen der Sowjet-Union: Deutsche Volkskunde im ausserdeutschen Osten: vier Vorträge. Berlin: Walter de Gruyter, 52-81.

Smirnitskaya, S. V. 2000: [V. M. Schirmunski und das Leningrader Zentrum der Erforschung der deutschen Siedlungen]. In: Nemtsy $v$ Rossii. Russko-nemetskie nauchnye i kul'turnye svyazi [Germans in Russia. Russian-German scientific and cultural relations]. St.Petersburg, 61-70.

Смирницкая, С. В. 2000: В. М. Жирмунский и Ленинградский центр по изучению немецких поселений в России. Hемџы в России. Русско-немеикие научные и культурные связи. СПб.

Svetozarova 2013: Svetozarova, N. D. [Reports on folkloristic and dialectological study travels by Viktor Žirmunskij as a unique source of information for Germanistics.]. Magister Dixit, 3 (09).

Светозарова, Н. Д. 2013: Отчеты об экспедициях как уникальный источник информации (на материале фольклорно-диалектологических экспедиций В. М. Жирмунского). Magister Dixit, №3 (09). https://cyberleninka.ru/article/n/otchety-ob-ekspeditsiyah- 
kak-unikalnyy-istochnik-informatsii-na-materiale-folklornodialektologicheskih-ekspeditsiy-v-m-zhirmunskogo (25.03.20).

Svetozarova 2015: Svetozarova, N. D. [Hermann Bachmann and his book «Durch die deutschen Kolonien des Beresaner Gebietes»]. St.Petersburg. Nestor-Istorija.

Светозарова Н. Д. 2015: Герман Бахман и его книга «Поездка в немецкие колонии Березанского района». СПб., НесторИстория. 2015

Weigun: Weigum David. Damals auf der Krim. Landsmannschaft der Deutschen aus Russland e.V . Stuttgart, s. a.

Zinder, L. R. 1998: [V. M. Schirmunski und die Inseldialektologie]. Yazyk $i$ Yazykovaya deyatel'nost' 1, 187-193.

Зиндер, Л. Р. 1998: В М. Жирмунский и островная диалектология. Язык и речевая деятельность 1, 187-193

Žirmunskaja, Starec, Naiditsch, Svetozarova 2016: Žirmunskaja A. V., Starec M. L., Naiditsch L. E, Svetozarova N. D. Hrsg. Die Welt der deutschen Kolonie. Fotos aus den folkloristisch-dialektologischen Forschungsreisen von V. M. Schirmunski. Sankt-Petersburg. NestorIstorija.

Жирмунская, А. В., Старец, М. Л., Найдич, Л. Э., Светозарова, Н. Д. 2016 (авт.-сост.). Мир немецкой колонии. Фотографии из фольклорно-диалектологических экспедищий $B$. М. Жирмунского. Санкт-Петербург: Нестор-История.

Žirmunskij 1933=2018: Zirmunskij, V. Ergebnisse und Aufgaben der mundart- und volkskundlichen Erforschung der deutschen Siedlungen in der UdSSR. In: Bertleff, Eckhard, Svetozarova, 2018: 167-199. First publication: Жирмунский В. М. Итоги и задачи диалектографического исследования немецких поселений СССР, Советская этнография, 1933, 2, 84-112.

Žirmunskij, V. M. 1929=1976: [Problems of the colonial dialectology]. In: Žirmunskij, V. M. 1976: Obshchee $i$ germanskoe yazykoznanie [General and German Linguistics]. Leningrad, 491-516.

Жирмунский В. М. Проблемы переселенческой диалектологии. В: Жирмунский В. М. 1976: Общее и германское языкознание. Л., 491-516.

Žirmunskij, V. M. 1932=1976: [Methods of the social geography]. In: Žirmunskij, V. M. 1976: Obshchee $i$ germanskoe yazykoznanie [General and German Linguistics]. Leningrad, 422-452.

Жирмунский, В. М. Методика социальной географии. В: Жирмунский В. М. Общее и германское языкознание. Л, 422452. 